Research Article

\title{
The Comparison between the Osteogenic Differentiation Potential of Clay-Polya- crylonitrile Nanocomposite Scaffold and Graphene-Polyacrylonitrile Scaffold in Human Mesenchymal Stem Cells
}

\author{
Fatemeh Sadat Tabatabaei Mirakabad ${ }^{1}$, Simzar Hosseinzadeh ${ }^{2}$, Hojjat Allah Abbaszadeh ${ }^{3}$, Maryam \\ Sadat Khoramgah ${ }^{1}$, Hossein Ghanbarian ${ }^{1}$, Javad Ranjbari ${ }^{1 ๓ \dagger}$, Bahram Kazemi ${ }^{1 \longleftarrow \dagger}$ \\ ${ }^{1}$ Department of Biotechnology, School of Advanced Technologies in Medicine, Shahid Beheshti University of Medical Science, \\ Tehran, Iran. \\ ${ }^{2}$ Department of tissue engineering and regenerative medicine, School of Advanced Technologies in Medicine, Shahid Beheshti \\ University of Medical Sciences, Tehran, Iran. \\ ${ }^{3}$ Department of Biology and Anatomical Sciences, School of Medicine, Shahid Beheshti University of Medical Sciences, Tehran, \\ Iran. \\ † These authors contributed equally \\ —Corresponding authors. E-mail: ranjbarijavad@sbmu.ac.ir; bahram_14@yahoo.com; f.taba123@yahoo.com; Tel: +98 21-22439848
}

Received: May 22, 2019; Accepted: Aug. 16, 2019; Published: Aug. 16, 2019.

Citation: Fatemeh Sadat Tabatabaei Mirakabad, Simzar Hosseinzadeh, Hojjat Allah Abbaszadeh, Maryam Sadat Khoramgah, Hossein Ghanbarian, Javad Ranjbari, and Bahram Kazemi, The Comparison between the Osteogenic Differentiation Potential of Clay-Polyacrylonitrile Nanocomposite Scaffold and Graphene-Polyacrylonitrile Scaffold in Human Mesenchymal Stem Cells. Nano Biomed. Eng., 2019, I I (3): 238-253.

DOI: 10.5101/nbe.v11i3.p238-253.

\begin{abstract}
Nowadays, bone repair by means of stem cells potential is considered as a new approach in regenerative medicine. Adipose-derived mesenchymal stem cells (AD-MSCs) have been investigated as a plentiful cell source with the ability of osteogenic differentiation which can play an important role in bone tissue engineering applications. Discovering proper elements in combination of scaffolds structure to stimulate osteogenesis in adipose-derived stem cells is one of the major concerns in this issue. Porous polymeric scaffolds such as polyacrylonitrile (PAN) and susceptible nanoparticles have attracted a lot of attention recently due to biodegradability and differentiation potential respectively. In the present study, clay-PAN nanocomposite (CPN) and graphene-PAN scaffold have been electrospuned separately and evaluated from the point of the osteogenic potential in AD-MSCs. The objective of this study was to determine the effect of clay and graphene nanoparticles with PAN nanofibers on the fate of viability and osteogenesis of AD-MSCs. First, isolated mesenchymal cells were characterized by flow cytometry. After cell culture on the surface of scaffolds MTT assay, scanning electron microscope (SEM) and DAPI staining were done. The scaffolds were characterized and osteogenic differentiation potential of AD-MSCs has been investigated. The results have indicated that alkaline phosphatase (ALP) activity, calcium content and collagen expression of cells which cultured on clay-PAN nanofibers were higher than cells which cultured on graphene-PAN scaffold. Taken together, these results suggest that porous nanofiber clay-PAN scaffold can enhance the osteogenic differentiation of AD-MSCs, and can be used as a new biodegradable scaffold for bone tissue engineering applications.
\end{abstract}

Keywords: Clay; Graphene; Polyacrylonitrile; Osteogenic differentiation; Mesenchymal stem cell 


\section{Introduction}

Nowadays in developed countries, people older than 50 years old suffer from musculoskeletal diseases which consist of a vast majority of chronic pain. According to the World Health Organization (WHO) official announcement of Bone and Joint Decade (2000-2010), recent developments in bone defect regeneration are in the center of attention in worldwide tissue engineering studies [1].

Adipose-derived mesenchymal stem cells (ADMSCs) have been used in different applications of tissue engineering in recent years. Multipotential ability, relative abundance and easy facility of harvest are advantages of these cells relative to other cells. Self-renewal potential of these cells enables researchers to increase the number of isolated AD-MSCs in a controlled manner at in vitro environment. In this way, the amount of tissue required during the initial extraction is limited, and continued cell expansion occurs after seeding on biomaterial scaffolds [2].

By providing both mechanical support and sites for cell interaction, tissue engineering scaffolds need to mimic the organization and function of the native extracellular matrix (ECM). Alternative ways have been established to improve cell-scaffold interactions. A porous network is required for ideal scaffold which causes appropriate mechanical strength of scaffold. The natural ECM of bone tissue consists of nanoscale ranges of collagen fibers type-I. These collagen fibril diameters is usually between 260-410 $\mathrm{nm}$, and through their orientation build a multifibril three-dimensional (3D) and porous network. Via the electrospinning techniques potentials, it is possible to make a 3D porous network of fibers with the same size scale diameters. For this purpose, electrospun nanofibrous structures are being used as scaffolds for tissue engineering nowadays. The electrospun structures have exceptional properties such as great specific surface area with high porosity and 3D interconnected pore networks which cause cell growth and proliferation on scaffolds. In this way, various biodegradable polymers of synthetic origin have been used to prepare nanofibrous scaffolds via the electrospinning technique. There is a wide interest in using synthetic polymers owing to their extraordinarily mechanical properties, controlled degradation rates and economic efficiency [2, 3]. Polyacrylonitrile (PAN) is an important engineering polymer material which is commonly used in agriculture, textiles, filtration, protective materials and pharmaceuticals due to its good thermal properties, chemical resistance and superior mechanical characteristic [4].

On the other hand, the unique properties of nanomaterials in tissue engineering have captured a great deal of attention as experimental tools in biomedical applications. A plenty of research has provided a strong evidence that nanostructures not only passively interact with stem cells but also actively engage and mediate these cells functions [5]. Clay minerals are naturally layered silicate constructions (phyllosilicates) that have crystallized to micro- and nanometer-sized particles. Clay nanoparticles have been used in porous polymer scaffolds to increase compressive strength and stiffness which are vital elements for scaffold design in tissue engineering, especially in bone repair [6, 7]. Clay nanoparticles have an important role in the structure to generate strong porous scaffolds with strength and stiffness equivalent to trabecular bone. These polymeric materials have the potential of minimally invasive delivery and the toughness characteristic of soft biological tissues, so they are useful in hard and soft tissue regeneration applications [8, 9]. Also some researches have studied the effects of clay nanoparticles to stimulate the osteogenic induction of responsive cells through direct clay-interactions. Additionally, the improvements in cell adhesion, proliferation, and differentiation due to the presence of clay nanoparticles demonstrated in some studies [8].

In addition to clay nanoparticle, graphene is interesting for the fabrication of functional nanocomposites because of its high surface-tovolume ratio and excellent mechanical, thermal, and electrical properties [10]. Graphene is a planar carbonbased sheet of $\mathrm{sp}^{2}$ hybridized carbon atoms that are compactly organized into a 2D hexagonal lattice. Also, graphene is biocompatible material which is suitable for diverse biomedical applications. Interestingly, graphene surfaces have an affinity with cells and can effect on cell proliferation subsequently. Moreover, the chemical nature of graphene seems to have a role in the differentiation of stemcells. The mentioned evidences confirmed the considerable potential of graphene as an element in tissue engineering scaffolds [11]. In this study, AD-MSCs were allowed to proliferate after seeding on two PAN-based-electrospun scaffolds which consist of clay and graphene nanoparticles. The comparison between two scaffolds were analyzed for their effects on AD-MSCs viability, proliferation, 
and osteogenic differentiation. Also, PAN electrospun scaffold and adipose-derived mesenchymal stem cells cultured on tissue culture polystyrene (TCPS) plates have served as control groups. Up to now, no study has been conducted to compare the effects of clay and graphene nanoparticles on the fate of cells seeded on the PAN-based-electrospun scaffolds. These kinds of studies are necessary to understand the positive effects of nanoparticles in electrospun scaffolds for the repair and regeneration of bone tissues and others. The goal of the present study was to determine the effects of these two scaffolds on proliferation and osteogenic differentiation of ADMSCs. So this study hypothesized that the clay-PAN nanocomposite (CPN) scaffold would accelerate ADMSCs osteodifferentiation more than graphene-PAN scaffold and increase alkaline phosphatase (ALP) and calcium deposition throughout the scaffold more, which indicates its potential to serve as a bone tissue engineering scaffold using AD-MSCs.

\section{Experimental Materials}

Poly acrylonitrile (PAN) copolymer and nanoclay (Montmorillonite clay) powder were purchased from Sigma-Aldrich, USA. PAN copolymer (Aldrich 2501441-9) average molecular weight was 150,000 Da. Graphene nanoplatelets (xGnP®) grade $\mathrm{C}$ powder was purchased from XG Sciences. PAN, nanoclay and nanographene were dissolved in dimethyl formamide (DMF, Sigma-Aldrich) in room temperature (RT). For cell culture, reagent such as Dulbecco's Modified Eagle's Medium (DMEM), fetal bovine serum (FBS), phosphate buffered saline (PBS), and trypsin/ ethylenediaminetetraacetic acid were purchased from Gibco, Germany.

\section{Methods \\ Fabrication of clay-PAN, graphene-PAN and PAN electrospun scaffolds}

Before electrospinning, for clay-PAN nanocomposite (CPN) scaffold fabrication, $0.14 \mathrm{~g}$ of clay powder was dissolved in $4 \mathrm{~mL}$ of DMF with 20 min sonication in warm water bath. Then $0.28 \mathrm{~g}$ of PAN was added to the above solution and stirred at $700 \mathrm{rpm}$ in $25{ }^{\circ} \mathrm{C}$ for $4 \mathrm{~h}$. For graphene-PAN scaffold fabrication, $0.28 \mathrm{~g}$ of PAN were dissolved in $3.5 \mathrm{~mL}$ of DMF. Then $0.05 \mathrm{~g}$ of $\mathrm{xGnP}$ powder was dissolved in $0.5 \mathrm{~mL}$ of DMF with 20 min sonication in warm water bath, added to the above solution, and then stirred well at $700 \mathrm{rpm}$ in 25 ${ }^{\circ} \mathrm{C}$ until it was solved completely. It is noteworthy that before this study, the biological properties of graphenePAN (by using $\mathrm{xGnP}$ powder) for tissue engineering and regenerative medicine had not yet been explored. PAN fabrication was done with $0.28 \mathrm{~g}$ of PAN powder which was dissolved in $4 \mathrm{~mL}$ of DMF and stirred at $700 \mathrm{rpm}$ in $25^{\circ} \mathrm{C}$ for $4 \mathrm{~h}$. Then, the obtained solutions was transferred to separated syringes and run with electrospinning device. Electrospinning (Nano Spinner, Iran) was performed at the voltage of $15 \mathrm{kV}$ throughout, and the distance between the collector and nozzle tip was adjusted at $15 \mathrm{~cm}$. In this way, the solvent had enough time to evaporate from the jet with a rotating drum speed of $400 \mathrm{rpm}$ and a collector which was wrapped with aluminum foil with the potential to collect the scaffold fibers. The flow rate of the ultimate solution was $0.3 \mathrm{~mL} / \mathrm{h}$. Finally, nanofibers were collected on the drum and used for characterization and cell culture process. For cell culture uses, the fabricated scaffolds were treated with $70 \%$ ethylalcohol under UV radiation for 20 min and washed three times with PBS. Then, the sterilized scaffolds were incubated overnight with DMEM and became ready for cell culture uses.

\section{Characterization of electrospun nanofibers}

Structure and morphology of synthesized scaffolds were analyzed by scanning electron microscope (SEM). Gold layer was coated on the surface of scaffolds and then observed via MIRA3 SEM (Tescan, Czech Republic). Also, Fourier transform infrared spectroscopy (FTIR) was obtained from ALPHA FTIR Spectrometer (Bruker) in the wavenumber range of $4000-400 \mathrm{~cm}^{-1}$ to recognize molecular components, structures and functional groups. PAN scaffold was used as control. To measure the surface hydrophilicity of clay-PAN, graphene-PAN and PAN nanofibers, scaffolds were cut $10 \times 10 \mathrm{~mm}$ and fixed on microscope slides to demonstrate the contact angles finally. In this way, $2 \mu \mathrm{L}$ of deionized water was located on the scaffold surface at RT, and the contact angle was measured in less than 1 min through a 15 plus OCA instrument (Data Physics, Germany). All final data were drop shaped, and were captured with charge-coupled device camera and analyzed by means of software. Also, the tensile test for evaluating the mechanical properties of electrospun nanofibers was done. The clay-PAN, graphene-PAN and PAN nanofibrous scaffolds were also evaluated for stressstrain response through a mechanical testing machine (Santam (Iran, SPM20)) at a $10 \mathrm{~mm} / \mathrm{min}$ crosshead 
speed. In summary, the thickness of electrospun samples was measured by means of a digital micrometer and the samples were cut into a rectangular shape of $10 \mathrm{~mm}$ wide and $50 \mathrm{~mm}$ length. Once the loading of samples reached $0.5 \mathrm{kN}$, the typical stressstrain response of electrospun mats was plotted as a stress-strain curve. Then, the results of the mechanical tests were shown as mean values and standard deviation. The measurements were obtained based on stress-strain curves of each sample and tensile strength, elongation and Young's modulus $(E)$ were calculated [12].

\section{Isolation and characterization of AD-MSCs}

Adipose-derived mesenchymal stem cells (ADMSCs) were isolated from human subcutaneous adipose tissues after liposuction surgery (Taleghani General Hospital, Tehran, Iran) based on the medical ethics committee guidelines of Ministry Health of IR. Isolated tissues were kept in a container of Hanks buffer salt solution (HBSS) with streptomycin and penicillin. Adipose tissues were cut by surgical blade after it was washed three times by phosphate buffer saline (PBS) (Sigma, USA). Then, the pieces of tissue treated with collagenase enzyme for digestion. So the fragmented pieces of tissue were incubated in DMEM (Invitrogen Co, USA) media which were supplemented by $0.2 \%$ collagenase I (Sigma, USA) with shaking at $37^{\circ} \mathrm{C}$ for $30 \mathrm{~min}$. Then, for $10 \mathrm{~min}$, samples were centrifuged at $1500 \mathrm{rpm}$, supernatants removed, cell pellets were suspended on DMEM with $15 \% \mathrm{FBS}$, and finally were incubated at $37^{\circ} \mathrm{C}$ in a humidified $5 \% \mathrm{CO}_{2}$ atmosphere in incubator to reach proper density. The media of the cells were exchanged every two days using DMEM culture media with $10 \%$ FBS. After suitable confluency of cells in passage 2, they were ready for use in tests. To confirm the obtained cells by evaluation of MSCs surface markers, flow cytometry analysis was used with special antibodies. To achieve this, fluorescent isothiocyanate (FITC)-conjugated mouse anti-human CD73, CD90, CD105 and CD11b, CD45 and CD34 were applied (Sigma-Aldrich, USA). In this way, the mesenchymal cells with 90-100\% confluency were washed and detached by addition of $0.25 \%$ trypsinEDTA. Then, the suspended cells with DMEM (10\% FBS) were precipitated through centrifugation (1500 rpm, $5 \mathrm{~min}$ ) and the supernatant was discarded. Next, the cells suspended in blocking solution and were kept undisturbed on the ice for $20 \mathrm{~min}$. Then, $50 \mu \mathrm{L}$ of cell suspended in blocking solution was added to each tube containing diluted antibody solutions and was mixed gently, and left undisturbed on ice for 20 min. Then, the cells were added with $3 \mathrm{~mL}$ of PBS, mixed, and precipitated by centrifugation (1500 rpm, $5 \mathrm{~min}$ ), and the supernatant was discarded. This step was repeated once for removing antibodies. Staining reagent was added to reaction tubes and allowed for 15 min on ice. Then $3 \mathrm{~mL}$ of PBS was added and the cells were precipitated by centrifugation (1500 rpm, $5 \mathrm{~min}$ ), and the supernatant was removed. $3 \mathrm{~mL}$ of PBS was again added to reaction tubes, and after the cells were suspended, the cells were precipitated by centrifugation (1500 rpm, $5 \mathrm{~min}$ ), and the supernatant was removed. This step was repeated once more for cell washing [13].

\section{Cytocompatibility and viability study}

Adipose-derived mesenchymal stem cells (ADMSCs) which were isolated from human adipose tissues were maintained at $37{ }^{\circ} \mathrm{C}$ in a humidified $5 \% \mathrm{CO}_{2}$ atmosphere in incubator, using DMEM culture media (Gibco, Germany) with 10\% FBS (Gibco, Germany) and 1\% penicillin. After suitable confluency of mesenchymal stem cells, the cells were washed by PBS and detached by trypsin/ ethylenediaminetetraacetic acid. Cytocompatibility of clay-PAN, graphene-PAN and PAN nanofiber scaffolds were assessed by seeding the mesenchymal stem cells on them. AD-MSCs were seeded at a density of $1 \times 10^{4}$ cell/well in 96-well. Viability assay was conducted by using 3-[4,5 dimethylthiazol- 2yl]-2,5diphenyl tetrazolium bromide (MTT) powder (SigmaAldrich, UK) on days 1, 3, 7, 14 and 21. In this way, the samples were incubated with MTT solution for 3 h. At this step, the sediments were soluble in dimethyl sulfoxide (Sigma-Aldrich, UK). Then, the absorbance of color solution was measured through Elisa reader (BioTek EL $\times$ 800) instrument at wavelengths of $570 \mathrm{~nm}$ where all experiments were performed in triplicates.

\section{Human mesenchymal stem cell adhesion studies}

AD-MSCs attachment to scaffolds studied through 4,6-Diamidino 2-phenylindole staining test and SEM images. In this way, scaffolds were cut by $0.5 \times 0.5$ $\mathrm{cm}^{2}$ and AD-MSCs were seeded at a density of $1 \times$ $10^{4}$ cell/well in three groups of scaffolds. After 7 and 21 days, DAPI staining was done. In this way, the samples were washed with $\mathrm{PBS}$ ( $\mathrm{pH} 7$ ) and incubated with paraformaldehyde 4\% for $10 \mathrm{~min}$ and washed with PBS for another time. Then 0.1\% Triton X-100 
were used for 2 min and washed with PBS. Next, 4,6-diamidino 2-phenylindole (DAPI) stain (SigmaAldrich, UK) was used to stain the cells' nuclei in the dark for 5 min. The samples were washed three times with PBS and kept in cold and dark places before taking photographs by a Nikon fluorescent microscope (Eclipse Terminal Emulator 2000-S, Japan) [14]. In addition, cells' attachment was approved by scanning electron microscope (SEM) images which were taken on day 21. In this way, the seeded scaffolds were washed twice with PBS and the cells were fixed on the scaffolds by $4.5 \%$ glutaraldehyde for $2 \mathrm{~h}$. In next step, the scaffolds were washed with PBS. Then, they were dipped in ethanol densities (60-100\%) and dried overnight to dehydrate the scaffolds. Finally, gold layer was coated on the surface of scaffolds and examined by Hitachi SEM (SU3500, Japan).

\section{Osteogenic markers of human mesenchymal stem cells studies}

The osteogenic differentiation was examined through common osteogenic markers such as alkaline phosphatase (ALP) activity, trichrome staining and calcium content assays. To measure ALP activity, the total protein of cells was extracted by using $200 \mu \mathrm{L}$ of RIPA lysis buffer at days 7, 14 and 21. Then, the lysate were centrifuged at $15000 \mathrm{rpm}$ at $4{ }^{\circ} \mathrm{C}$ for $15 \mathrm{~min}$, and the ALP activities of supernatant were evaluated using p-nitrophenyl phosphate (pNPP) as a phosphatase substrate (ALP Kit, Pars Azmoon Iran) at $450 \mathrm{~nm}$. The obtained enzyme activity level was normalized against total protein [15].

Masson's staining of the seeded scaffolds was also performed as routine histological protocols to quantify the collagen secreted in the process of osteogenic differentiation as an indirect index. In this way, samples were fixed in $4 \%$ paraformaldehyde, embedded in paraffin, and sectioned in to $5 \mu \mathrm{m}$ sections. The sections were prepared for histological analysis by staining with Masson's trichrome. First, sections were deparaffinized and rehydrated through alcohol and washed gently. Sections were stained in Weigert's iron hematoxylin working solution for 10 min and then washed in distilled water. Then, sections were stained in Biebrich scarlet-acid fuchsin solution for 10-15 min and washed gently. Sections were placed in phosphomolybdic-phosphotungstic acid solution for 10-15 min. At last, sections were transferred directly to aniline blue solution stain for 5-10 min, rinsed briefly and placed in 1\% acetic acid solution for 2-5 min. Then they were washed, dehydrated and mounted for microscopic analysis which demonstrated the collagen fibers via blue stain. For determination of the percentage area of collagen, a custom ImageJ macro, based on a color deconvolution technique, was used [16-18].

For evaluation of calcium deposited amount and other elements in the scaffold-seeded stem cells, the samples were examined by scanning electron microscopy (SEM, JSM-7600F; JEOL Ltd., Tokyo, Japan) with energy-dispersive X-ray spectroscopy (EDS) (JEOL Ltd., Tokyo, Japan) to perform EDS mapping at days 7 and 21. EDS can make images known as digital mapping, which are compositional map of the samples [19].

\section{Statistical analysis}

In this study, statistical analysis was performed by SPSS statistical software. Student's t-test was used to evaluate differences between results of electrospun scaffolds and the $\mathrm{p}$ value of less than or equal with 0.05 and 0.01 were interpreted as being noteworthy. The whole data are exposed in curve areas as mean \pm standard error (SD).

\section{Results and Discussion \\ Characterization of electrospun nanofibers analysis}

Imaging of electron microscopy of scaffolds as demonstrated in Fig. 1 shows that they were reticular and consisted of random and homogenous nanofibers. The average diameter of the clay-PAN, graphene-PAN and PAN nanofibers of scaffolds were $100 \pm 14 \mathrm{~nm}$ (SD), $230 \pm 14 \mathrm{~nm}$ (SD) and 150 $\pm 18 \mathrm{~nm}$ (SD), respectively. Dispersion of clay and graphene nanoparticles were effective because agglomerates cannot be found with SEM images. The results of SEM images as shown in Fig. 1 showed that the scaffolds are composed of suitable nanofibers diameters and structures which are well appropriate to prepare condition for guiding stem cells to bone tissue engineering.

Based on FTIR results (Fig. 2), bands at 3000-4000 $\mathrm{cm}^{-1}$ demonstrated $\mathrm{OH}$ vibration and absorption area in $2243 \mathrm{~cm}^{-1}$ was showed the nitrile bonds in all PANbased scaffolds [20]. The characteristic peaks of $\mathrm{Na}^{+}-$ MMT in clay-PAN nanocomposite (CPN) scaffold, observed at 3614 and $1038 \mathrm{~cm}^{-1}$, which are ascribed to the $\mathrm{OH}$ stretching of the lattice water, $\mathrm{Si}-\mathrm{O}$ and $\mathrm{Al}-$ $\mathrm{O}$ stretching. Also, the peaks of PAN are observed at 

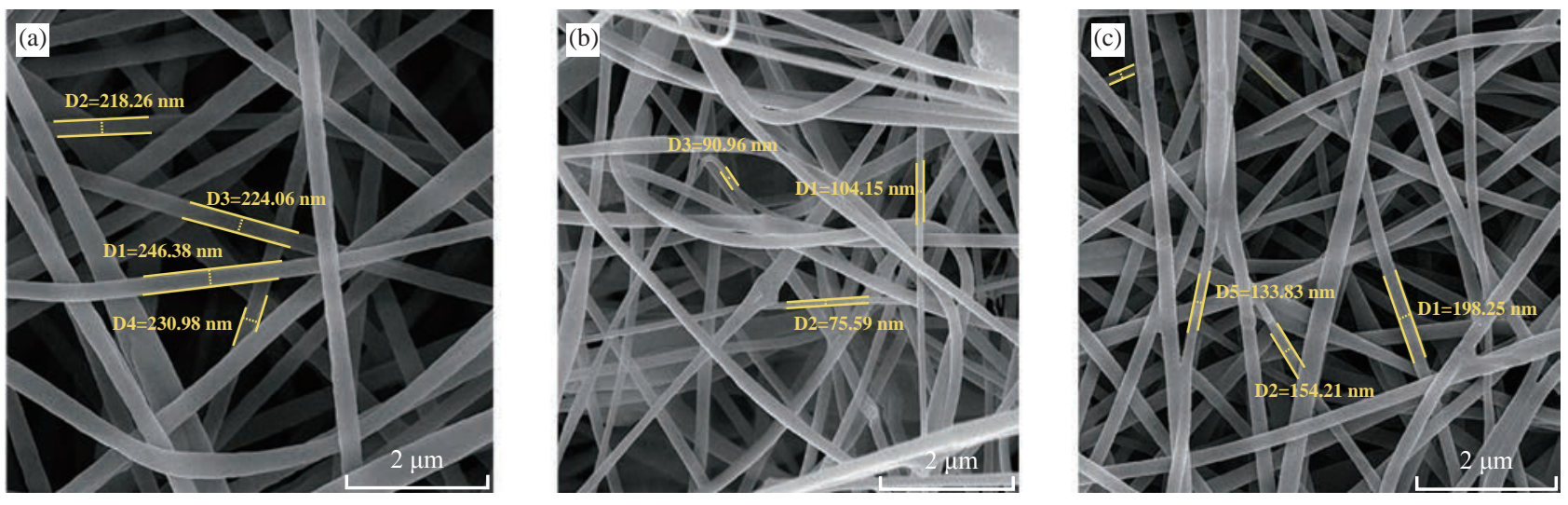

Fig. 1 SEM homogenously micrograph of (a) graphene-PAN, (b) clay-PAN and (c) PAN nanofiber electrospun nanofiber scaffolds with $2 \mu \mathrm{m}$ scale bar.

the bands at 1661, 1449, 1360, 1252 and $759 \mathrm{~cm}^{-1}$, corresponding to quinoid ring structure, benzenoid ring structure, and out of-plane bending, respectively [21]. Also in graphene-PAN scaffold, FTIR analyses demonstrated weak peaks near $1724 \mathrm{~cm}^{-1}$ and 1351 $\mathrm{cm}^{-1}$ which indicate the doping state of graphene-PAN and a broad band near $3500 \mathrm{~cm}^{-1}$ describes the $\mathrm{N}-\mathrm{H}$, C$\mathrm{H}$ and hydroxyl group stretching vibrations [22].

It is clear that contact angle demonstrates the wettability of the material surface, indicating hydrophilic/hydrophobic features of the material. In general, a contact angle above 90 corresponds to a hydrophobic surface, while a contact angle value under 90 represents a hydrophilic surface. So the amount of hydrophilicity of scaffolds were measured through contact angle. Contact angle of water was measured in less than one minute. As can be seen in Fig. 3, the scaffolds could maintain hydrophilicity characteristics, as one of the positive points of scaffolds. The results showed that the contact angle of CPN scaffold was $30.226^{\circ}$, the contact angle of graphene-PAN scaffold was $47.310^{\circ}$ and the contact angle of PAN scaffold was $51.169^{\circ}$. These results showed that the hydrophilic properties of the surface of CPN were richer than graphene-PAN and PAN scaffolds, which made the CPN as a more suitable site for attachment of cells.

Mechanical properties of scaffolds were evaluated by tensile test. Table 1 shows the related results of tensile examination of the prepared clay-PAN, graphene-PAN and PAN electrospun nanofibrous scaffolds. The scaffold membrane with graphene nanosheets demonstrated an obvious increase in tensile strength, modulus and elongation compared to the CPN and PAN scaffold. So the addition of graphene nanoparticles can improve the crystallinity of scaffold nanofibers due to the increase in the mobility of polymeric chains. As there are fewer functional groups on the surface of graphene nanoparticles compared to the CPN scaffold, fewer bonds like hydrogen bonding between graphene nanoparticles and polymer chains are formed, leading to greater mobility of polymer chains.

\section{Mesenchymal stem cell characterization}

AD-MSCs were characterized after isolation at the passage 2 via flow cytometrey by evaluation of mesenchymal related surface markers. Flow cytometric analysis results are shown in Fig. 4. It shows that in some tubes, the amount of fluorescent dye bound to the cell surface was greater than that of the negative control, and in some tubes, the amount of fluorescent dye bound to the cell surface was similar to that of the negative control. The red curve demonstrated the isotype control and the blue curve showed the intended CD markers. These results indicated that the human adipose derived mesenchymal stem cells were positive for CD73, DC90 and CD105, and were negative for

Table 1 Tensile properties of nanofibrous membranes

\begin{tabular}{|c|c|c|c|}
\hline Sample & Max tensile strength (MPa) & Tensile modulus & Break tensile strain (\%) \\
\hline Clay-PAN & $0.35 \pm 0.1$ & $0.04 \pm 0.1$ & $15 \pm 1.2$ \\
\hline Graphene-PAN & $2.7 \pm 0.6$ & $0.44 \pm 0.1$ & $21 \pm 1.7$ \\
\hline PAN & $1.3 \pm 0.4$ & $0.15 \pm 0.1$ & $11 \pm 1.5$ \\
\hline
\end{tabular}



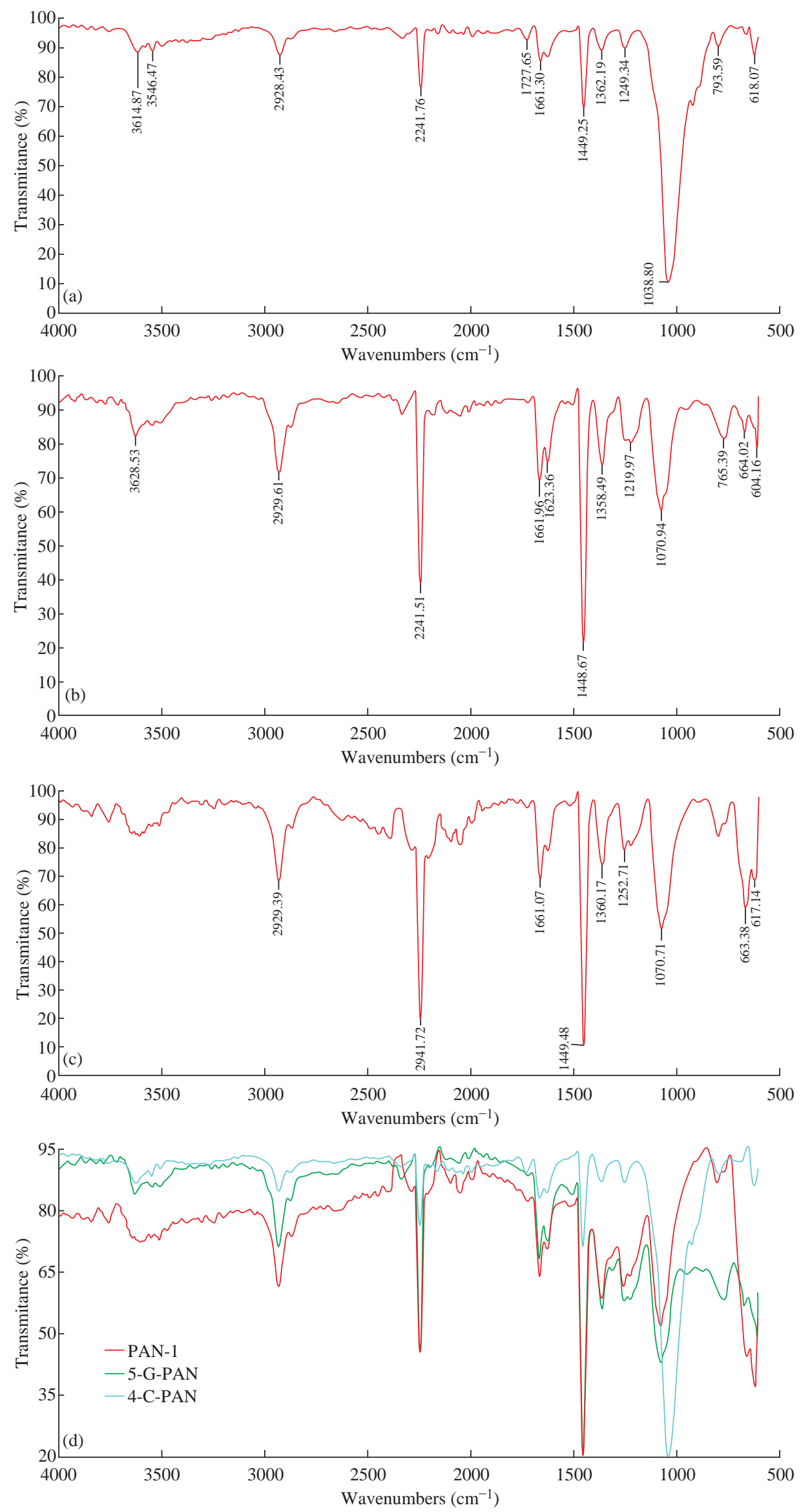

Fig. 2 FTIR spectra of (a) clay-PAN nanocomposite scaffold, (b) graphene-PAN scaffold, (c) PAN scaffold, and (d) clay-PAN, graphene-PAN and PAN scaffold together. 


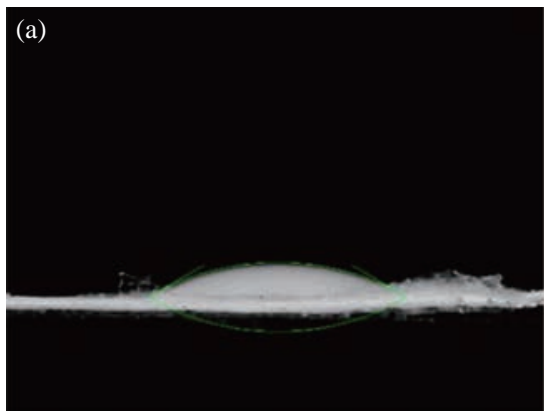

Contact angle: $30.226^{\circ}$

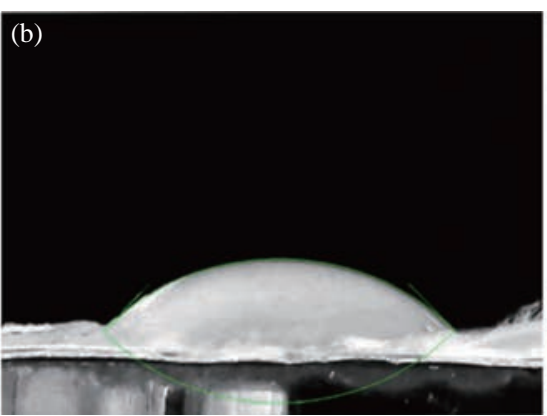

Contact angle: $47.310^{\circ}$

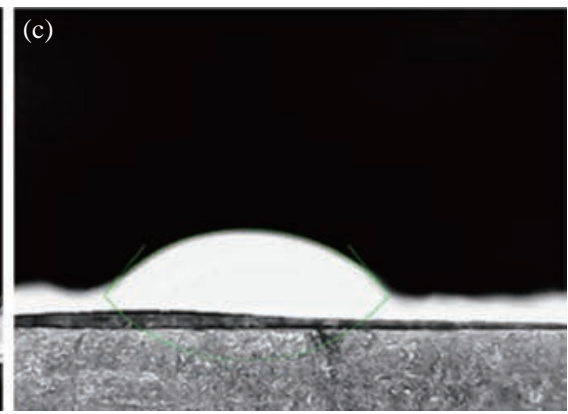

Contact angle: $51.169^{\circ}$

Fig. 3 Contact angle micrograph of (a) clay-PAN nanocomposite, (b) graphene-PAN and (c) PAN scaffold.

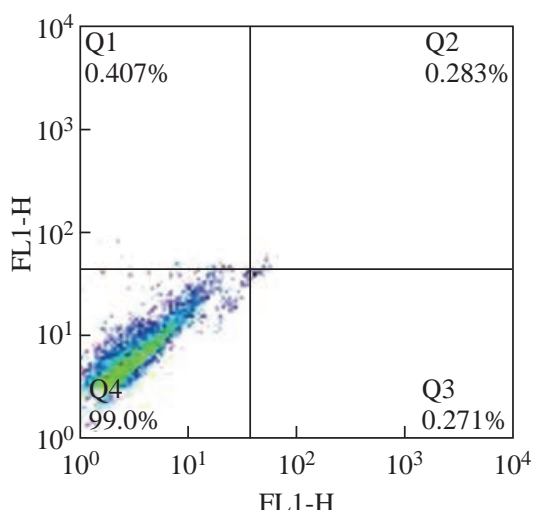

CD45-FITC/CD34-PE

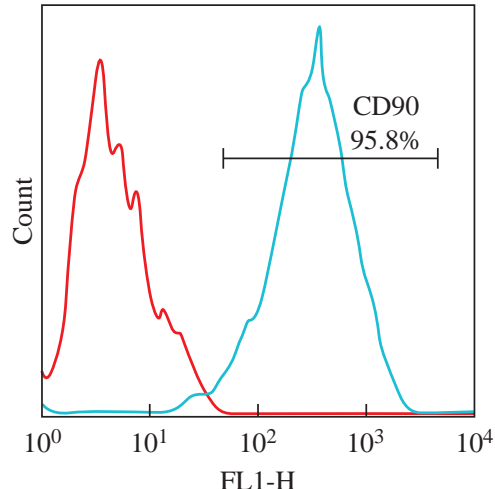

CD90

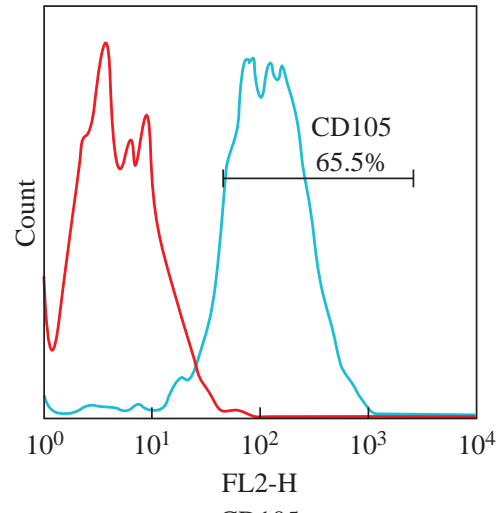

CD105

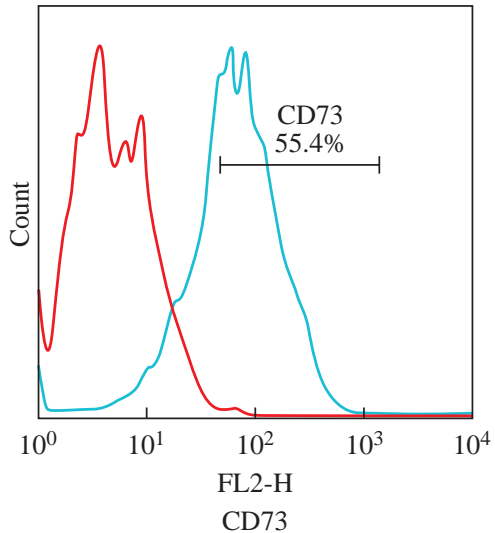

CD73

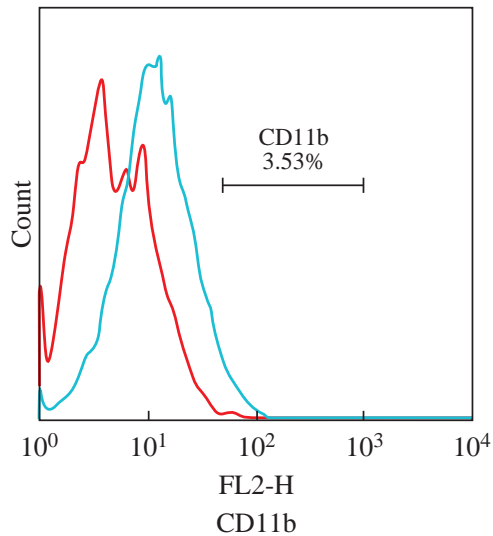

CD11b

Fig. 4 Flow cytometry analysis of isolated AD-MSCs. Results are negative for CD11b, CD45 and CD34 and positive for CD73, CD90 and CD105.

\section{CD11b, CD45 and CD34.}

\section{Cytocompatibility and viability study analysis}

MTT analysis of cultured cells on prepared scaffolds was performed in order to investigate the biocompatibility of prepared scaffolds. The cellular viability of scaffolds was estimated via colorimetric assay (MTT assay) at $570 \mathrm{~nm}$. The obtained results demonstrated absorbance values (mean and standard deviation) after 1, 3, 7, 14 and 21 days as showed in Fig. 5. The comparison between the absorbency values of scaffolds exposed significant differences between
clay-PAN nanocomposite (CPN) and graphene-PAN scaffold with control groups (PAN scaffold and TCPS) over cell culture $(p<0.05)$ (Fig. 5).

\section{Human mesenchymal stem cell adhesion studies analysis}

Attachment of AD-MSCs to scaffolds studied through DAPI staining test and SEM images. To study the differentiated cells' nuclei on scaffolds and show the cells on the surface of clay-PAN, graphenePAN and PAN nanofibrous scaffolds, AD-MSCs were cultured on the scaffolds and evaluated after 7 and 
21 days through DAPI staining test. PAN scaffold was considered as control group. Images obtained by using fluorescence microscopy can be seen in Fig. 6, representing the adhesion of cells with healthy nucleus to the scaffolds which was shown by transparent blue color in all three scaffolds.

The results of SEM images (Fig. 7) showed that AD-MSCs adhered well to graphene-PAN, clay-PAN and PAN scaffolds, and that they developed cell-cell connection due to the suitability of the surface of the scaffolds. On the other hand, clay-PAN nanocomposite (CPN), graphene-PAN and PAN scaffolds provide a suitable microenvironment for cell-cell and cell-matrix interaction. So, SEM images revealed that human mesenchymal stem cells cultured on all three scaffolds were attached, growth and spread on the surface of the scaffolds during 21 days. Also, these data confirmed the biocompatibility test.

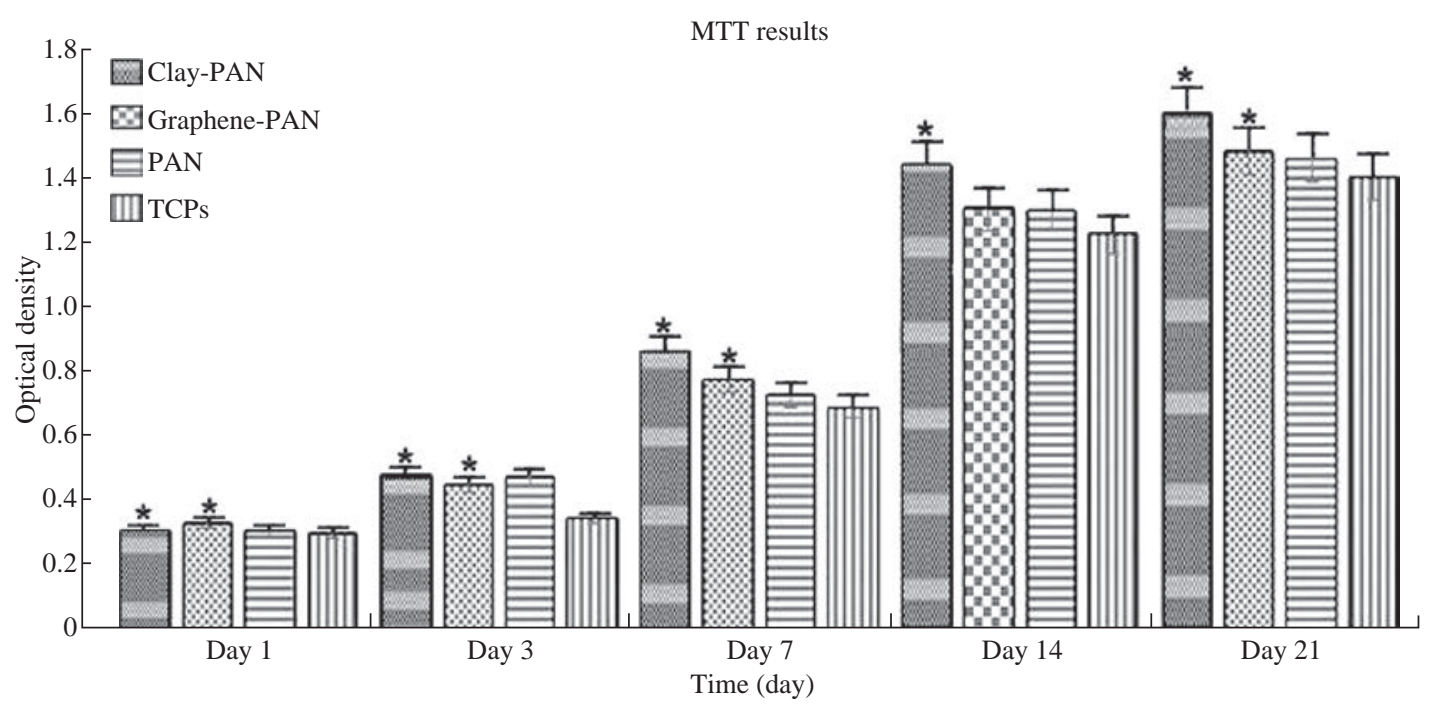

Fig. 5 MTT results of electrospun nanofibrous scaffolds.
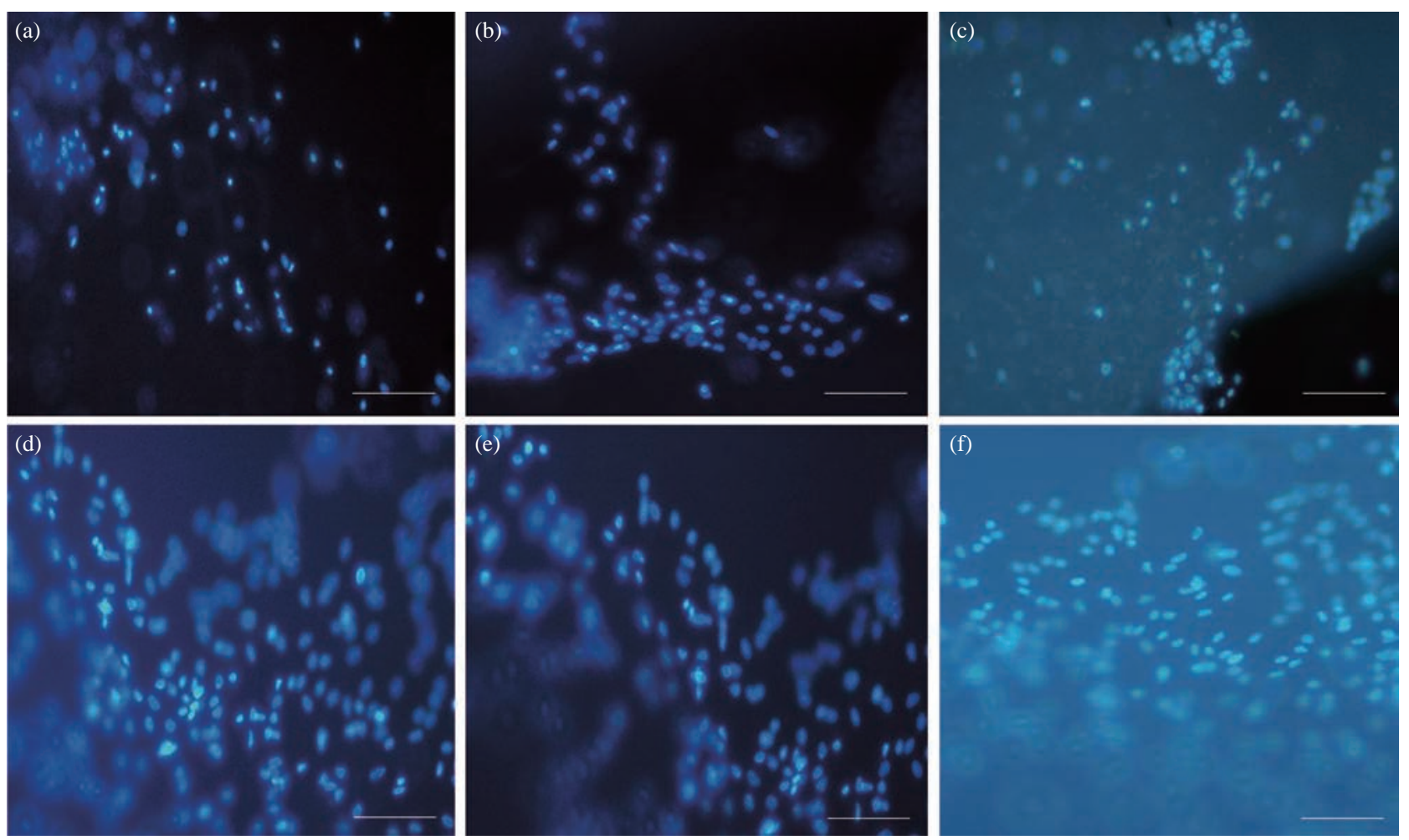

Fig. 6 Images of DAPI staining after culture of AD-MSCs on (a) clay-PAN nanocomposite scaffold after 7 days, (b) graphene-PAN scaffold after 7 days, (c) PAN scaffold after 7 days, (d) clay-PAN nanocomposite scaffold after 21 days, (e) graphene-PAN scaffold after 21 days, and (f) PAN scaffold after 21 days with microscope magnification $20 \times$. 

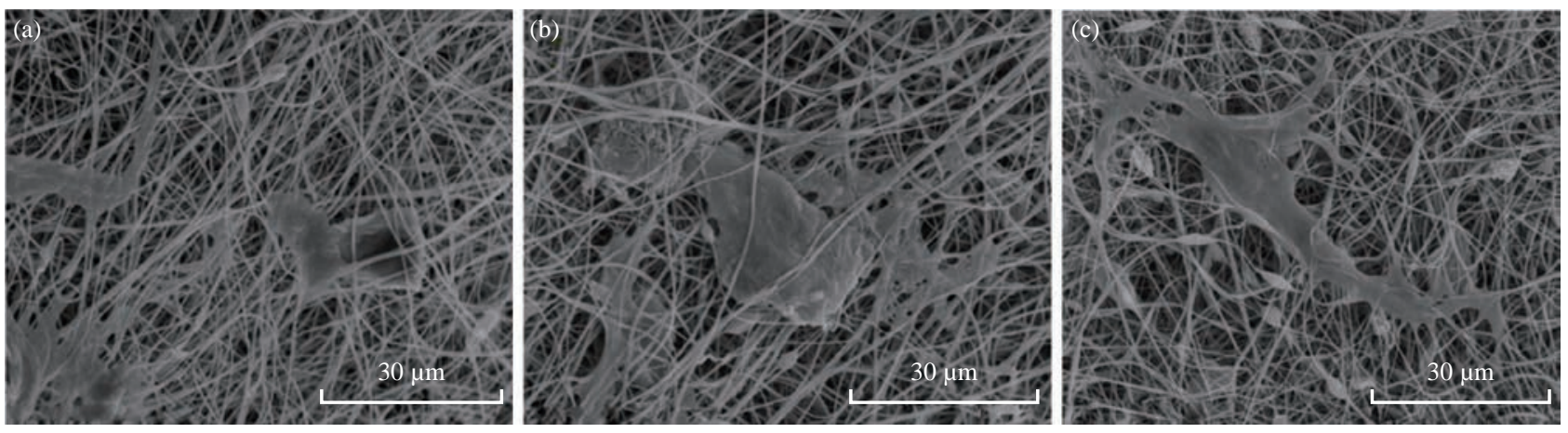

Fig. 7 Surface morphology of the electrospun scaffolds after AD-MSCs culture on the scaffolds after 21 days. (a) Graphene-PAN nanofiber scaffold; (b) clay-PAN nanocomposite scaffold; and (c) PAN nanofiber scaffold with scale bar $30 \mu \mathrm{m}$.

\section{Alkaline phosphatase activity, trichrome staining and calcium content}

ALP activity was evaluated during osteogenic differentiation of AD-MSCs cultured on clay-PAN nanocomposite (CPN), graphene-PAN and PAN scaffolds at days 7, 14 and 21, as shown in Fig. 8. The pattern of ALP activity had a higher activity at day 14, and clay-PAN scaffold had the highest ALP activity as compared with graphene-PAN scaffold and control groups (PAN scaffold and TCPS). All data exposed highly significant differences between CPN and graphene-PAN scaffolds with control groups over cell culture ( $p<0.05$, Fig. 8 ).

Masson's trichrome staining allowed the collagen deposition rate to be visualized as blue-stained tissue in 7 and 21 days in all groups (Fig. 9). In the medullary bone-depositing stage, collagen was secreted to form collagen fibers along with the bone deposition, and the collagen fibers subsequently connected to create collagen nets to form the basic framework for bone formation. If it will be continued, with the increase in secreted collagen, collagen fibers gradually merged to form the tabular structure as the mineralization matrix. In this study, the proportions of collagen in the graphene-PAN scaffold were $7.66 \pm 0.5 \%$ and $22.33 \pm 0.4 \%$ in 7 and 21 days post cell culture, and those in the clay-PAN nanocomposite (CPN) scaffold were comparatively $11.67 \pm 0.4 \%$ and $30.67 \pm 0.5 \%$. Also, the proportions of collagen in the PAN scaffold which served as control group was $2.01 \pm 0.5 \%$ and $5.11 \pm 0.5 \%$ after 7 and 21 days. As the most important component of bone tissue, collagen progressively increased with bone formation, and the discrepancy between groups revealed that the AD-MSCs on CPN scaffold produced 4 to $8 \%$ more collagen than the cells on graphene-PAN scaffold, and 9 to $25 \%$ more collagen than the cells on PAN scaffold, which indirectly indicates that bone formation was increased and more rapid in the clay-PAN scaffold ( $p<0.05$, Fig. $10)$.

Mineralization, as one of the most important late markers of osteogenic differentiation, was also investigated at days 7 and 21 (Fig. 11; Table 2). An increasing trend of calcium deposition was detected

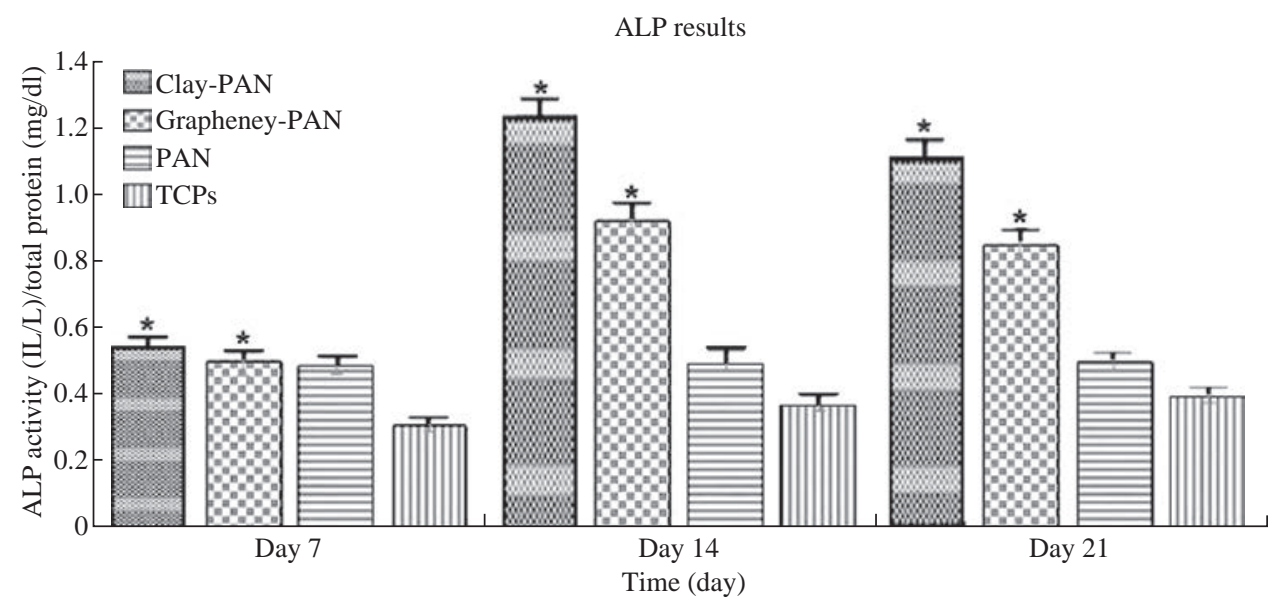

Fig. 8 ALP results of electrospun nanofibrous scaffolds. 

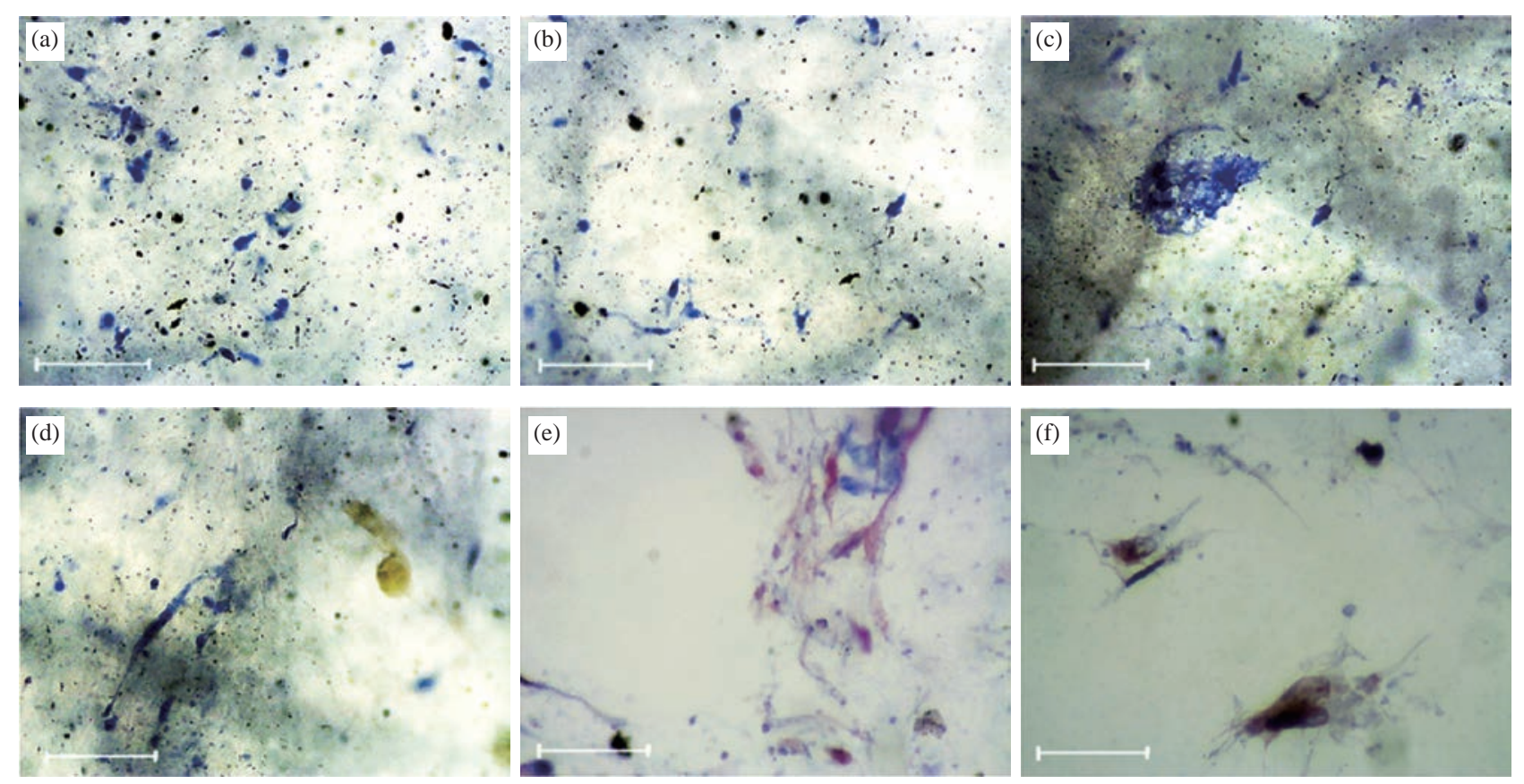

Fig. 9 Masson's trichrome staining of (a) clay-PAN nanocomposite in day 21, (b) clay-PAN nanocomposite in day 7, (c) graphenePAN scaffold in day 21, (d) graphene-PAN scaffold in day 7, (e) PAN scaffold in day 21, and (f) PAN scaffold in day 7 with scale bar $100 \mu \mathrm{m}$.

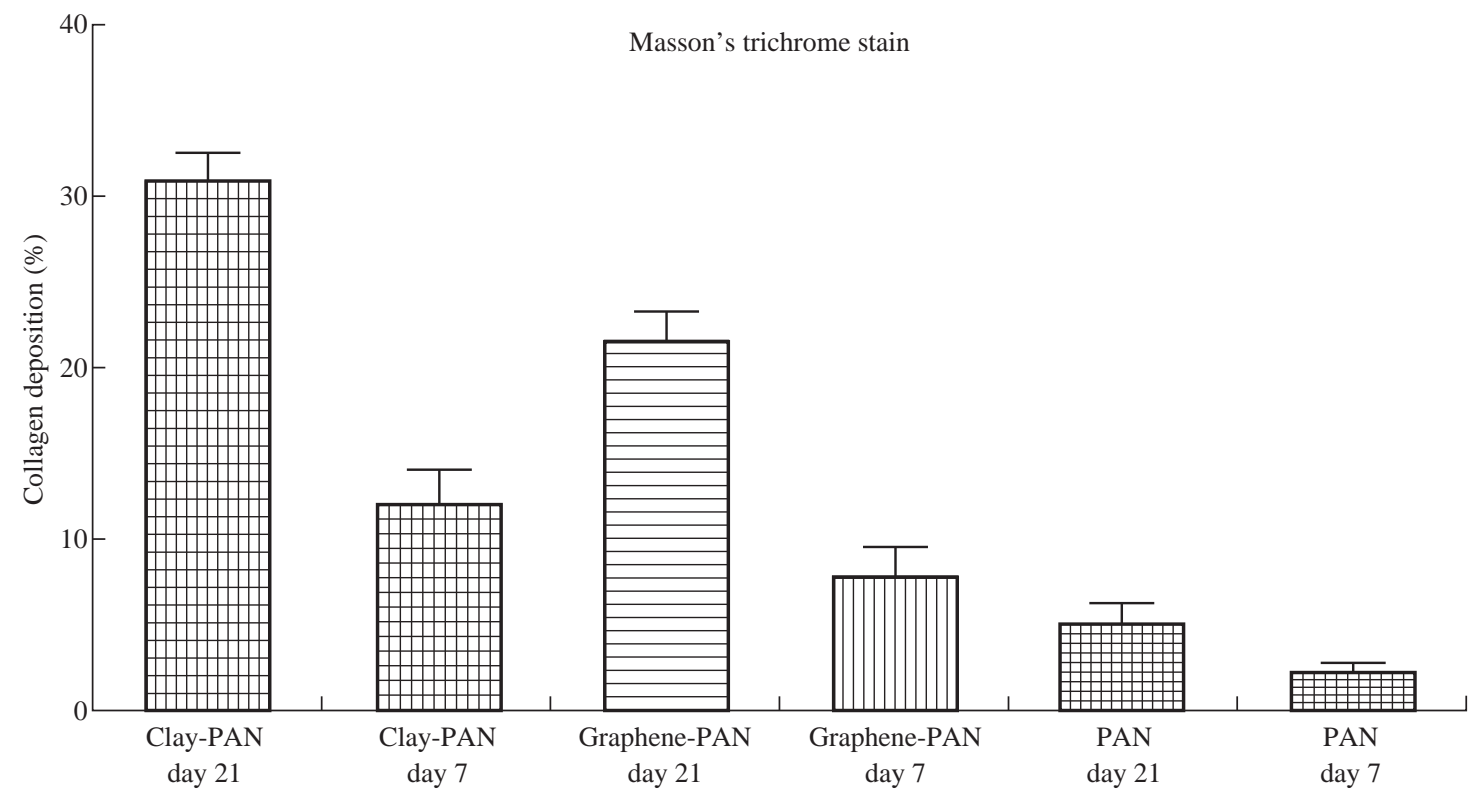

Fig. 10 The percentage area of collagen deposition in clay-PAN nanocomposite, graphene-PAN scaffold and PAN scaffold after hADSCs culture at 7 and 21 days with Masson's trichrome staining.
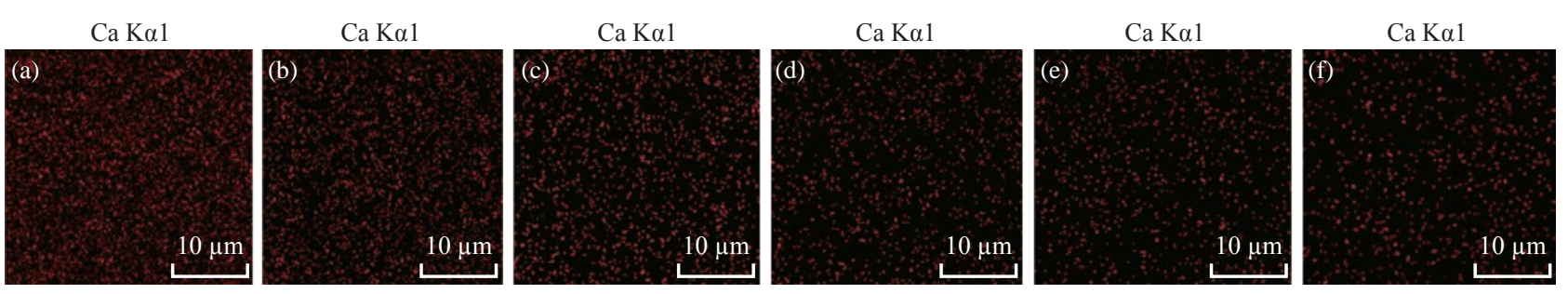

Fig. 11 Images of calcium deposition which was taken with SEM-EDS elemental mapping after culture of AD-MSCs on (a) clayPAN nanocomposite scaffold after 21 days, (b) clay-PAN nanocomposite scaffold after 7 days, (c) graphene-PAN scaffold after 21 days, (d) graphene-PAN scaffold after 7 days, (e) PAN scaffold after 21 days, and (f) PAN scaffold after 7 days with microscope magnification $100 \times$. 
in seeded cells on PAN, graphene-PAN and clay-PAN nanocomposite (CPN) respectively, which specified with bright spots in Ca maps (Fig. 11) and represented the Ca deposition in osteogenic differentiation. Also, through the EDS analysis the highest concentration of calcium observed in the CPN scaffold at days 7 and 21 (39.6 $\pm 0.8 \%$ and $52 \pm 0.6 \%$ ). Furthermore, the multielemental EDS results demonstrated the amount of $\mathrm{Ca}$, $\mathrm{Si}, \mathrm{Al}$ and $\mathrm{O}$ in samples (Table 2). It should be noted that $\mathrm{Si}, \mathrm{Al}$ and $\mathrm{O}$ elements were seen in CPN scaffold diagram due to the presence of these elements in clay nanoparticles basically (Table 2).

Table 2 Multi-elemental EDS results of nanofibrous membranes before and after culture of AD-MSCs on days 7 and 21

\begin{tabular}{lcccc}
\hline \multicolumn{1}{c}{ Sample } & $\mathrm{Ca}$ & $\mathrm{Si}$ & $\mathrm{Al}$ & $\mathrm{O}$ \\
\hline Clay-PAN, Day 7 & 39.6 & 241.7 & 131.9 & 68.6 \\
Clay-PAN, Day 21 & 52 & 241.7 & 96.9 & 65.8 \\
Graphene-PAN, Day 7 & 10.5 & 22.2 & 24.3 & 17 \\
Graphene-PAN, Day 21 & 13.4 & 22 & 25.1 & 14.8 \\
PAN, Day 7 & 7.7 & 1.5 & 1.7 & 10.8 \\
PAN, Day 21 & 3.7 & 2.1 & 2.3 & 5.7 \\
Clay-PAN scaffold & 2.4 & 83.9 & 25.6 & 11.3 \\
Graphene-PAN scaffold & 1.5 & 1.7 & 1.1 & 4.1 \\
PAN scaffold & 4 & 2.4 & 2.4 & 7.3 \\
\hline
\end{tabular}

\section{Discussion}

Designing of a scaffold depends on its performance and ultimate purpose. Assessment of a threedimensional scaffold needs to be conducted for cell proliferation aimed at its efficiency ultimately to replace a damaged tissue. Scaffold not only allows attachment of the cells but may also simplify cell migration, relocation biochemical factors, the release of nutrients elements, waste and materials produced through the cells, and imitation in vivo manner or mimic an environment biological implant to repair whole body tissues. To achieve these exceptional properties, the scaffolds should have definite structural potentials which it accessible through electrospinning method. In this way nanofibers produce with top touch biomimetic to ECM and play an important role in proliferation and differentiation of cells. This technique has some remarkable advantages such as great surface area to volume ratio, fluency of material combination, creation of different nanofiber structures, superficial functionalization, nanoscale fibers and organized porosity structure production [20]. Since the fibrils of the extra cellular matrix of bone tissue are also randomly organized and have nanometer-scale diameters, the electrospun fibers applied in this study are similar as in size to what cells may encounter with it in native tissue. For instance, Ye et al. [24] synthesized three-dimensional nanofibrous scaffolds via electrospining method. These electrospuned scaffolds have both nanofibrous morphologies and interconnected pores which were fabricated for the promotion of osteogenic differentiation of stem cells and bone regeneration [2, 23, 24]. The results from this study are significant in that they show that further optimization of the nanofibrous structures for bone tissue engineering can be achieved through the addition of nanoparticles to the scaffolds solution. As it approved in medicine, nanoparticles have been applied in many kinds of application which is one of the most significant ones is tissue engineering of scaffolds. Using scaffolds and nanoparticles together is a new approach in regenerative medicine for repairing defects $[25,26]$. For example Shafiei et al. electrospun a kind of layered double hydroxide/poly ( $\varepsilon$-caprolactone) nanocomposite scaffold and applied it for adipogenic differentiation of adipose-derived mesenchymal stem cells [27]. In another study which focused on bone differentiation, Karimi et al. applied Baghdadite nanoparticle - coated with poly L-lactic acid (PLLA) ceramics electrospun scaffold which can improved osteogenic differentiation of adipose tissue - derived mesenchymal stem cells [1]. Also in a study which was done by Ma et al. polymer electrospun scaffolds coated with hydrophilic hematite nanoparticles $(\alpha \mathrm{FeNPs})$ constructed to create a bioactive interface between the cells and scaffolds and increase the osteoinduction capacities of the scaffolds [28]. In the present study, the presence of clay and graphene nanoparticles in PAN-based scaffolds were approved with positive effects on uniformity of scaffolds and lead to bone differentiation of AD-MSCs which were seeded on nanofiber scaffolds. However, the clay-PAN nanocomposite (CPN) scaffold ability in bone differentiation of AD-MSCs was better than graphenePAN due to more suitable condition which it provide for stem cells. Better bone differentiation of CPN scaffold was approved via the significant results of ALP assay, trichrome stainig and calcium deposition as described in former sections. Actually, the clay nanoparticle elements act as a source of osteoinductive silica species which lead to the enhanced osteogenic responses in clay-PAN scaffold [8]. So, the innovative nanoclay scaffold could have improved osteoinduction capacities with better performance in bone tissue engineering. In previous studies, researchers studied 
clay and graphene nanoparticles effects on bone differentiation separately. For instance, Gibbs et al. showed the bone induction by clay nanoparticle gels at physiological doses of BMP [29]. Based on this study, it is clear that the addition of nanoclay in the PAN scaffold lead to higher ALP activity, calcium deposition and collagen deposition in AD-MSCs after 2-3 weeks of culture which is one of the most important sign of bone differentiation. Also Zhai et al. used nanoclay which was incorporated with polyethylene-glycol diacrylates and demonstrated that this biocompatible and biodegradable nanocomposite hydrogel is appropriate for stimulating in vitro and in vivo osteogenesis [30]. Moreover, Villaça et al. synthesized a kind of clay-polymer nanocomposite membrane containing sodium alendronate and leading to osteogenic activity in Saos-2 cells as a model of human osteoblasts [31]. Another study done by Song et al. demonstrated that cultured mesenchymal stem cells and PC12 cells on the polycaprolactone/graphene oxide scaffolds caused the cell adherence with growth and $0.3 \%$ and $0.5 \%$ concentration of graphene oxide improved the differentiation of mesenchymal stem cells into osteogenic cells [32]. Shuai et al. used polyvinyl alcohol and graphene oxide scaffolds for bone tissue engineering. They manufactured interconnected porous nanocomposite by means of laser sintering. The graphene oxide added improved the proliferation and differentiation of osteoblast like cells and the addition of $2.5 \mathrm{wt} \%$ of graphene oxide enhanced the strength of the scaffolds [33]. Like the study of Shuai which showed the addition of graphene oxide could increase the strength of the scaffolds, this study also showed that all parameters of tensile test for graphene-PAN scaffold such as tensile strength, modulus and elongation were higher than CPN and PAN scaffold. However, it is the first time that such a study has been done by using $\mathrm{xGnP}$ as a graphene source of PAN based scaffold and checked the in vitro biological properties. In addition, it is the first investigation that has compared the effects of clay and graphene nanoparticles in a polymeric-based scaffold and demonstrate the preferred scaffold for bone differentiation. In fact, in none of the studies conducted to date, the superiority of the clay nanoparticle to graphene nanoparticle has been shown in in-vitro or invivo environments. As a result of this study, CPN scaffold is better than graphene-PAN scaffold for ADMSCs differentiation into osteogenic cells. Briefly in this study, the whole structure of clay-PAN, graphene-
PAN and PAN scaffolds were assessed by SEM, FTIR, contact angle and tensile test afterward they fabricated by electrospinning technique. SEM images demonstrated the reticular and homogenous nanofibers. The average diameter of the clay-PAN, graphene-PAN and PAN nanofibers of scaffolds were $100 \pm 14 \mathrm{~nm}$

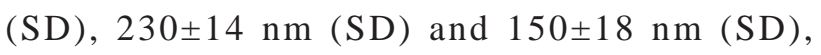
respectively, and dispersion of clay and graphene nanoparticles was effective in both scaffolds. Based on FTIR results, the characteristic peaks of $\mathrm{Na}^{+}-\mathrm{MMT}$ in CPN scaffold, specific peaks of graphene-PAN scaffold and specific peaks of PAN scaffolds were observed. Also, contact angle which demonstrates the wettability of the material surface, indicating hydrophilic or hydrophobic features of all scaffolds. Based on the results of this study the scaffolds had hydrophilic properties of the surface which is more in CPN scaffold and made it a suitable area for attachment of cells. Then the mechanical properties of scaffolds were evaluated by tensile test which demonstrated an obvious increase in tensile strength, modulus and elongation of graphene-PAN scaffold compared to the clay-PAN and PAN scaffolds. In next step, AD-MSCs isolated successfully and the cells approved through flowcytometry which were CD73+, CD90+, CD105+ and CD11b-, CD45-, CD34-. Then the isolated cells cultured on the top of clay-PAN, graphene-PAN and PAN scaffolds. The mesenchymal stem cells have been attached to the scaffolds and the potential of the fabricated scaffolds to conduct osteogenesis on seeded AD-MSCs have been assessed during 21 days. The biocompatibility of scaffolds for AD-MSCs were evaluated by MTT assay and the attachment of cells were evaluated by SEM images and DAPI staining. Biochemical tests have been applied to assess osteogenic differentiation. ALP is an enzyme which indicate the cell mineralization in the way of osteogenic differentiation of primary cells. The results of this study have indicated that the amount of ALP activity has been increased in AD-MSCs that were cultured on the whole scaffold groups through osteogenic differentiation from day 1 to 14 and formerly it has been decreased. This finding is in accordance with the role of ALP in osteogenic differentiation exactly in that ALP which is anticipated to have the crucial role and the maximum amount in mid-differentiation at day 14 [11]. Although the difference between ALP enzime concentrations was important in all time section at day of 7, 14, and 21, the highest concentration was observed in day 14 . 
According to the results of this study, ALP activity in CPN scaffold on days 14 and 21 was always higher than that of graphene-PAN and PAN scaffolds. These data were confirmed further by Mansson's trichrome staining and calcium deposition analysis. The superiority of CPN scaffold for osteogenic differentiation of AD-MSCs was approved on days 7 and 21 in Mansson's trichrome staining. This staining allowed the expression of collagen to be visualized as blue-stained tissue in all groups. Based on previous studies in the medullary bone-depositing stage, collagen was secreted to form collagen fibers along with the bone deposition. As the most important component of bone tissue, collagen progressively increased with bone formation, and the discrepancy between the scaffold groups revealed that the ADMSCs on CPN scaffold produced 4 to $8 \%$ more collagen than the cells on graphene-PAN scaffold and 9 to $25 \%$ more collagen than the cells on PAN scaffold. In addition, in calcium deposition analysis, the amount of $\mathrm{Ca}^{2+}$ deposition has been increased in cells cultured on the surface of all scaffolds groups through osteogenic differentiation from day 7 to 21 . On day 21, the highest amount of calcium was detected in cells cultured on CPN scaffold. This is the main factor for osteogenesis process. The results of this study have been demonstrated the effectiveness of the designated scaffold in the late stages of osteogenesis. Therefore, the current study represents the higher capability of clay nanoparticles in bone tissue engineering. In conclusion, the results of this study have revealed that biological behavior and osteogenic differentiation of AD-MSCs were improved more on CPN scaffold rather than graphene-PAN and PAN scaffolds. Although, further questions remain about the long-term fate of clay and graphene nanoparticles in scaffolds in in vivo assays for bone regeneration in critical-sized bone defects. These consist of the probability and impact of their cellular uptake, their ability for dissolution within the intercellular and intracellular spaces and the significance of particle size and surface area in consequence of the cytotoxicity of nanoparticles. Also clay and graphene nanoparticle effects on the fate of other kinds of cells in different types of scaffolds can be studied in future investigations. Thus, substantial work is still required to demonstrate the long-term biocompatibility of the clay and graphene particles before clinical application.

\section{Conclusions}

This study demonstrated for the first time, bone differentiation of AD-MSCs on clay-PAN, graphenePAN and PAN nanofiber electrospun scaffolds performance in vitro without adding any growth factors. The scaffold structures were approved by means of SEM images, FTIR analysis, contact angle and tensile test. After cell culture on scaffolds, the cell attachment was assessed with DAPI staining and SEM images; the scaffolds' biocompatibility was studied through MTT assay. Finally, ALP activity, Mansson's trichrome staining and calcium deposition were performed for all scaffolds which confirmed ADMSCs differentiation to osteogenic cells. The results of this study showed that the scaffolds indicated their good support for proliferation of the cells, but in all the mentioned tests clay-PAN Nanocomposite (CPN) scaffold demonstrated better results and significant advantages in comparison with graphene-PAN and PAN scaffolds in the same condition, which is due to the presence of clay nanoparticle in the CPN structure. Thus, this study can open new insights into fabrication of clay-based scaffolds with better compatibility to mimic the extracellular matrix of bone tissue. It supports the feasibility of using clay-based bicomponent nanofibers as a scaffolding material to promote bone formation. So the corresponding scaffolds can be introduced as a more promising candidate for bone repair.

\section{Acknowledgements}

The authors thank the Department of Medical Biotechnology, School of Advanced Technologies in Medicine, Shahid Beheshti University of Medical Science, for all the support provided. This is a part of report from a thesis entitled "Optimization of topography and surface properties of clay-PAN scaffold via nonoclay concentrations and investigation of its effects on bone differentiation of human mesenchymal stem cells.”, registered in Shahid Beheshti University of Medical Sciences.

\section{Conflict of Interests}

The authors report no declarations of interest. The authors alone are responsible for the content and writing of the paper. 


\section{References}

[1] Z. Karimi, E. Seyedjafari, F.S. Mahdavi, et al., Baghdadite nanoparticle - coated poly l - lactic acid (PLLA) ceramics scaffold improved osteogenic differentiation of adipose tissue - derived mesenchymal stem cells. Journal of Biomedical Materials Research Part A, 2019, 107A: 1284-1293..

[2] C.M. Haslauer, A.K. Moghe, J.A. Osborne, et al., Collagen-PCL sheath-core bicomponent electrospun scaffolds increase osteogenic differentiation and calcium accretion of human adipose-derived stem cells. Journal of Biomaterials Science, Polymer Edition, 2011, 22(13): 1695-1712.

[3] I. Bartolozzi, S. Cometa, C. Errico, et al., Multiblock polyurethanes based on biodegradable amphiphilic poly ( $\varepsilon$-caprolactone)/poly (ethylene glycol) segments as candidates for tissue engineering applications. Nano Biomedicine and Engineering, 2011, 3(2): 86-94.

[4] Y. Xiao, Y. Cao, R. Xin, et al., Fabrication and characterization of electrospun cellulose/polyacrylonitrile nanofibers with $\mathrm{Cu}$ (II) ions. Cellulose, 2018, 25(5): 29552963.

[5] X.Q. Liu, R.Z. Tang, Biological responses to nanomaterials: understanding nano-bio effects on cell behaviors. Drug Delivery, 2017, 24(2): 1-15.

[6] O.M. Istrate, B. Chen, Porous exfoliated poly ( $\varepsilon$-caprolactone)/clay nanocomposites: Preparation, structure, and properties. Journal of Applied Polymer Science, 2012, 125(S1): E102-E112.

[7] J. Calabria-Holley, S. Papatzani, B. Naden, et al., Tailored montmorillonite nanoparticles and their behaviour in the alkaline cement environment. Applied Clay Science, 2017, 143: $67-75$.

[8] J.I. Dawson, R.O. Oreffo, Clay: New opportunities for tissue regeneration and biomaterial design. Advanced Materials, 2013, 25(30): 4069-4086.

[9] MGA-A. Al-Mosawy, E.A.J. Al-Mulla, and M.J. Mohamad, Bentonite-based nano organic clay using chalcone and azo dye as organophilic reagents. Nano Biomed Eng., 2017, 9(2): 124-128.

[10] M. Silva N.M. Alves, and M.C. Paiva, Graphene polymer nanocomposites for biomedical applications. Polymers for Advanced Technologies, 2018, 29(2): 687700.

[11] S. Aznar-Cervantes, J.G. Martínez, A. Bernabeu-Esclapez, et al., Fabrication of electrospun silk fibroin scaffolds coated with graphene oxide and reduced graphene for applications in biomedicine. Bioelectrochemistry, 2016, 108: 36-45.

[12] S.I. Jeong, I.D. Jun, M.J. Choi, et al., Development of electroactive and elastic nanofibers that contain polyaniline and poly (l - lactide-co-e-caprolactone) for the control of cell adhesion. Macromolecular Bioscience, 2008, 8(7): 627-637.

[13] K. Imagawa, Method for producing pluripotent stem cells derived from dental pulp. Google Patents, 2018.

[14] M. Mahmoudifard, M. Soleimani, S. Hatamie, et al., The different fate of satellite cells on conductive composite electrospun nanofibers with graphene and graphene oxide nanosheets. Biomedical Materials, 2016, 11(2): 025006.

[15] M. Izadpanahi, E. Seyedjafari, E. Arefian, et al., Nanotopographical cues of electrospun PLLA efficiently modulate non-coding RNA network to osteogenic differentiation of mesenchymal stem cells during BMP signaling pathway. Materials Science and Engineering: C,
2018, 93: 686-703.

[16] G. GoMori, A rapid one-step trichrome stain. American Journal of Clinical Pathology, 1950, 20(7_ts): 661-664.

[17] C. Cunha, C.R. Almeida, M.I. Almeida, et al., Systemic delivery of bone marrow mesenchymal stem cells for in situ intervertebral disc regeneration. Stem Cells Translational Medicine, 2017, 6(3): 1029-1039.

[18] S.M. Lee, I.K. Park, Y.S. Kim, et al., Physical, morphological, and wound healing properties of a polyurethane foam-film dressing. Biomaterials Research, 2016, 20(1): 15.

[19] H.H. Chang, C.L. Cheng, P.-J. Huang, et al., Application of scanning electron microscopy and X-ray microanalysis: FE-SEM, ESEM-EDS, and EDS mapping for studying the characteristics of topographical microstructure and elemental mapping of human cardiac calcified deposition. Analytical and Bioanalytical Chemistry, 2014, 406(1): 359-366.

[20] M. Mohamadali, S. Irani, M. Soleimani, et al., PANi/ PAN copolymer as scaffolds for the muscle cell - like differentiation of mesenchymal stem cells. Polymers for Advanced Technologies, 2017, 28(9): 1078-1087.

[21] M.A. El-Ghaffar, A. Youssef, and A.A. El-Hakim, Polyaniline nanocomposites via in situ emulsion polymerization based on montmorillonite: Preparation and characterization. Arabian Journal of Chemistry, 2015, 8(6): 771-779.

[22] L. Yin, J. Wang, F. Lin, et al., Polyacrylonitrile/graphene composite as a precursor to a sulfur-based cathode material for high-rate rechargeable Li-S batteries. Energy \& Environmental Science, 2012, 5(5): 6966-6672.

[23] W.J. Li, C.T. Laurencin, E.J. Caterson, et al., Electrospun nanofibrous structure: a novel scaffold for tissue engineering. Journal of Biomedical Materials Research, 2002, 60(4): 613-621.

[24] K. Ye, D. Liu, H. Kuang, et al., Three-dimensional electrospun nanofibrous scaffolds displaying bone morphogenetic protein-2-derived peptides for the promotion of osteogenic differentiation of stem cells and bone regeneration. Journal of Colloid and Interface Science, 2019, 534: 625-636.

[25] Y.H. Alimohammadi, S.W. Joo. PLGA-based nanoparticles as cancer drug delivery systems. Asian Pac J Cancer Prev, 2014, 15: 517-535.

[26] P. Habibovic, K. de Groot, Osteoinductive biomaterials properties and relevance in bone repair. Journal of Tissue Engineering and Regenerative Medicine, 2007, 1(1): 2532.

[27] S.S. Shafiei, M. Shavandi, G. Ahangari, et al., Electrospun layered double hydroxide/poly ( $\varepsilon$-caprolactone) nanocomposite scaffolds for adipogenic differentiation of adipose-derived mesenchymal stem cells. Applied Clay Science, 2016, 127: 52-63.

[28] S. Ma, Z. Wang, Y. Guo, et al., Enhanced osteoinduction of electrospun scaffolds with assemblies of hematite nanoparticles as a bioactive interface. International Journal of Nanomedicine, 2019, 14: 1051.

[29] D. Gibbs, C. Black, G. Hulsart-Billstrom, et al., Bone induction at physiological doses of BMP through localization by clay nanoparticle gels. Biomaterials, 2016, 99: 16-23.

[30] X. Zhai, C. Hou, H. Pan, et al., Nanoclay incorporated polyethylene-glycol nanocomposite hydrogels for stimulating in vitro and in vivo osteogenesis. Journal of Biomedical Nanotechnology, 2018, 14(4): 662-674.

[31] J.C. Villaça, L.C.R. da Silva, K. Adilis, et al., Development and characterization of clay-polymer 
nanocomposite membranes containing sodium alendronate with osteogenic activity. Applied Clay Science, 2017, 146: 475-486.

[32] J. Song, H. Gao, G. Zhu, et al., The preparation and characterization of polycaprolactone/graphene oxide biocomposite nanofiber scaffolds and their application for directing cell behaviors. Carbon, 2015, 95: 1039-1050.

[33] C. Shuai, P. Feng, C. Gao, et al., Graphene oxide reinforced poly (vinyl alcohol): nanocomposite scaffolds for tissue engineering applications. RSC Advances, 2015,
5(32): 25416-25423.

Copyright $(\subset$ Fatemeh Sadat Tabatabaei Mirakabad, Simzar Hosseinzadeh, Hojjat Allah Abbaszadeh, Maryam Sadat Khoramgah, Hossein Ghanbarian, Javad Ranjbari, and Bahram Kazemi. This is an open-access article distributed under the terms of the Creative Commons Attribution License, which permits unrestricted use, distribution, and reproduction in any medium, provided the original author and source are credited. 